Relations industrielles

Industrial Relations

\title{
Les camps de travaux forcés en pays communistes
}

\section{François Brégha}

Volume 6, numéro 2, mars 1951

URI : https://id.erudit.org/iderudit/1023232ar

DOI : https://doi.org/10.7202/1023232ar

Aller au sommaire du numéro

Éditeur(s)

Département des relations industrielles de l’Université Laval

ISSN

0034-379X (imprimé)

1703-8138 (numérique)

Découvrir la revue

Citer cet article

Brégha, F. (1951). Les camps de travaux forcés en pays communistes. Relations industrielles / Industrial Relations, 6(2), 48-52. https://doi.org/10.7202/1023232ar

Tous droits réservés @ Département des relations industrielles de l’Université Laval, 1951
Ce document est protégé par la loi sur le droit d'auteur. L’utilisation des services d'Érudit (y compris la reproduction) est assujettie à sa politique d'utilisation que vous pouvez consulter en ligne.

https://apropos.erudit.org/fr/usagers/politique-dutilisation/ 
cliques, de façon à stimuler ou à décourager, selon le cas, les placements privés et à maintenir ainsi à un niveau stable l'emploi et le revenu national.

Par contre, pour que cette stabilisation ne s'opère pas à un niveau trop bas, et que ne soit pas compromise, pour autant, la prospérité générale, la théorie comporte qu'on s'applique à assurer aux classes populaires, qui ont le plus de propension à consommer, des revenus qui leur permettent de maintenir une demande suffisante sur le marché des biens et des services.

Le moyen prévu, à cette dernière fin, consiste d'une part dans le dégrèvement des impôts indirects dont l'incidence est générale; et, d'autre part, dans le financement, par l'impôt direct progressif mettant à contribution les épargnes improductives, d'un programme compréhensif de sécurité sociale dont les prestations, en augmentant directement ou indirectement le pouvoir d'achat des masses, doivent assurer une utilisation maximum des forces productives.
L'incidence économique qu'on attend de ces programmes de sécurité sociale dans une politique générale de stabilisation de l'économie de marché à un haut niveau d'emploi et de revenus, ne doit pas cependant nous faire oublier qu'ils sont d'abord inspirés par des préoccupations sociales, par le souci d'assurer aux classes économiquement faibles, ce supplément de bien-être qu'elles réclament et que, même dans une économie de plein emploi, elles ne peuvent s'assurer par leur travail.

Nous tenant aux limites d'une interprétation sociologique, nous espérons avoir démontré que le nouveau régime, avec la conception politicoéconomique dont il s'inspire, est le produit normal de l'Etat libéral du XIXième siècle: qu'il est né d'une part des insatisfactions et de l'insécurité produites par l'application du libéralisme économique; d'autre part, de l'incorporation des masses populaires à l'Etat démocratique dans la ligne de développement du libéralisme politique.

\title{
Les camps de travaux forcés en pays communistes
}

\author{
par Françors BrEghA, de l'Institut tchécoslovaque d l'étranger
}

Dans cette seconde étude, nous essaierons de préciser les raisons réelles de l'établissement des camps de travaux forcés en pays communistes, d'après ce que nous en savons et d'après nos sources d'information.

Les faits cités dans l'étude précédente prouvent sans aucun doute que les CTF ne sont nullement un moyen d'éducation des oisifs, mais bien un moyen de terreur illégal employé par le groupe au pouvoir contre tout le peuple tchécoslovaque. Leur but est, d'une part, la suppression des éléments dangereux ou simplement de ceux dont l'attitude envers le régi- me n'est pas positive, et, d'autre part, l'intimidation de la masse de la population qui pourrait se montrer éventuellement hostile au régime. Ce sont là des raisons générales de l'établissement des camps. Il y a pourtant des raisons directes, immédiates; elles sont, d'une part, d'ordre politique et d'autre part, d'ordre économique.

\section{Raisons politiques}

Les raisons d'ordre politique de l'établissement de la terreur au sein d'un état dictatorial réside dans le 
principe même de la dictature. Ce principe établit que la volonté de lorganisation politique au pouvoir (ou,celle du dictateur) est plus forte que la volonté de l'Etat, exprimée par les lois. De ce fait, là dictature, représentée par le groupe au pouvoir, constitue un état illégal, dans lequel l'ordre légal est violé par ceux mêmes qui, détenant le pouvoir, devraient imposer l'observation des normes qu'ils ont établies.

En effet, la loi ne représente pas seulement un moyen pour le groupe au pouvoir de réaliser. sa volonté, mais encore, elle réserve au citoyen une certaine sphère d'action libre (aussi restreinte que possible), dans laquelle il est son propre maître et peut guider ses actions, et disposer de son temps. Et c est précisément cette sphère non contrôlée que la loi, même la plus rigide, ne peut enchaîner complètement; qui peut constituer la base de départ, pour l'individu, d'un effort tendant à renverser la situation sociale et politique qui lui est défavorable. La dictature ne peut pas faire autrement que d'essayer d'abolir même ce petit reste de liberté, parce que cela pourrait lui être dangereux; et elle le fait en comblant cette lacune par un procédé illégal. C'est ainsi que la dictature est obligée de violer les lois qu'elle a établies elle-même pour éviter toute opposition qui, dans le cadre purement légal; pourrait menacer son existence.

Par conséquent, la dictature est un système de force et de brutalité, comme le constate d'ailleurs expressément le théoricien du totalitarisme, Lenine: « La conception scientifique de la dictature n'est rien d'autre qu'une conception illimitée qui n'est liée par aucune loi et par aucune règle, d'un pouvoir qui repose directemeni sur la force. ${ }^{1}$

(1) Staline, Les Questions du leninisme, p: 111 (édition tchèque).
Tout régime totalitaire s'impose des tâches dont l'accomplissement n'est possible qu'en collaboration avec la nation toute entière. Ces tâches consistent, pour les régimes commu: nistes, dans l'établissement du com. munisme dans le monde entier.

Il va de soi qu une telle tâche exigè le déploiement maximum des forces de chaque citoyen, et que l'Etat communiste est obligé de punir ou menacer non seulement ses adversaires, mais encore tous ceux qui ne montrent pas assez d'enthousiasme et d'es: prit de sacrifice dans laccomplissement de ce premier devoir de chaque citoyen des états communistes. Par conséquent, le régime s'efforce d'isoler ses adversaires, de les supprimer et de gagner les autres citoyens à sa cause. Et tout régime totalitaire sait que son programme s'écroulerait si la nation y faisait opposition. Privés de la possibilité d'exprimer librement leurs opinions, les combattants pour la liberté se constituent dans les organisations non-politiques, si de telles organisations sont abolies, ils se constituent illégalement. Si les organisations illégales sont elles-mêmes écrasées, ils travaillent individuellement, chacun pour soi, et deviennent une force invisible, impalpable, insaisissable, contre laquelle l'Etat ne peut rien: il ne lui reste qu'à recourir à la terreur.

C'est pourquoi la terreur et sa conséquence inévitable, les camps de concentration, est inséparable de chaque régime dictatorial. Les CTF dans les états communistes ne sont pas propres seulement à un certain pays ou à une certaine situation; ils sont une conséquence inévitable de l'établissement du régime communis. te, ils dérivent de l'essence même de ce régime. Sans eux, le régime. s'écroulerait. C'est pourquoi, partout où le régime communiste apparait, son avènement est suivi d'un établissement de ces camps. 
Telle est la raison générale qui rend inévitable l'établissement, sous quelque forme que ce soit, des camps de concentration pour les adversaires réels ou présumés du régime dictatorial. Mais dans les états dominés par le Parti communiste, il existe encore une raison spéciale (bien que dérivée des raisons générales) nécessitant cet établissement.

La doctrine communiste énonce comme condition indispensable de la victoire mondiale du communisme, l'oppression totale de tous les groupes sociaux qui pourraient menacer la classe au pouvoir - ou prétendue au pouvoir - et particulièrement le prolétariat. C'est l'issue de la lutte des classes qui forme la base de toute la politique communiste. Par conséquent: liquidation impitoyable de la petite et moyenne entreprise qui n'a pas été détruite par la nationalisation; liquidation progressive des journaux démocratiques et de leurs dirigeants, lutte impitoyable contre toute organisation, contre tout reste d'une organisation non-communiste (comme l'organisation des gymnastes - Sokol, l'organisation de jeunesse non-communiste, l'organisation d'etudiants), si elles ne sont pas encore soumises à la volonté du Parti; enfin, attaque contre l'ennemi le plus obstiné et le mieux organisé: l'Eglise.

Cette lutte ne peut être menée par des moyens légaux en raison de cette * lacune de la loi dont il a déjà été question. Un cordonnier ou un tailleur ne peuvent pas être punis - selon la loi - parce qu'ils sont propriétaires d'une petite entreprise étatisée. Nul, non plus, ne peut être puni légalement, parce qu'il n'est pas membre du Parti, parce qu'il ne participe pas aux brigades de travail ou parce qu'il fréquente l'église. Cette lacune est assez importante pour empêcher le régime de poursuivre sa politique de collectivisation et sa politique de lutte des classes.

Ce sont les CTF qui doivent com- bler cette lacune. Il existe des faits pour lesquels aucune loi ne prévoit une punition ou un emprisonnement. On déclare donc que le travail dans un CTF n'est pas une punition; c'est seulement un stage dans une école ou un centre d'éducation, permettant aux rééduqués de rentrer dans la vie et la société en « socialistes sincères et convaincus $\gg$.

\section{Raisons économiques}

Les raisons d'ordre économique sont plus complexes, mais aussi plus visibles que les raisons politiques. Elles peuvent être classées d'après:

a) la situation du marché du travail,

b) le mouvement abstentionniste et

c) la situation financière du gouvernement.

\section{a) Situation du marché du travail}

L'économie planifiée, surtout quand elle doit aboutir à l'industrialisation du pays et à la préparation d'une gigantesque machine de guerre, avec ses conséquences importantes: fusion des entreprises, diminution des « intermédiaires 》 (dans le commerce surtout), engendrent des chan. gements profonds sur le marché du travail. Or, une économie planifiée est impossible sans une transformation du marché du travail et par conséquent sans les mesures susceptibles de le modifier par la force.

Le régime communiste en Tchécoslovaquie ayant essayé de résoudre ce problème par la répartition du travail, a complètement échoué à cause de la résistance passive du peuple qui refuse de s'incliner devant les ordres du Parti communiste, considérant ce régime comme un régime dictatorial qui lui est hostile et lui est imposé par une puissance étrangère. Les résultats des efforts du Gouvernement communiste en Tchéroslovaquie sont tragiques pour lui. En voici un exemple pour l'année 1948: 
Nombre de personnes embuuchées par mesures officielles
Nombre de personnes

qui sont demeurées

dans ce nouvel emploi

Mines

54,167

Hauts fourneaux

18,953

Métallurgie

121,644

21,915

Chimie

Ceramique

34,205

( (et 3,880 départs parmi les anciens employés)

3,400

22,500

2,800

3,100

En face de cette situation, le régime communiste est inévitablement obligé de recourir à la force; au lieu de répartir le travail objectivement, il emploie la force contre ses adversaires politiques, vrais ou supposés, en les enfermant dans les CTF et en se créant ainsi une armée de main-d'oeuvse dont il peut disposer à son gré, et qui est obligée de faire les travaux les plus pénibles et les plus dangereux dans les mines, les carrières, les haut-fourneaux, etc.

\section{b) Mourement abstentionniste}

Ce mouvement prend un aspect de plus en plus menaçant dans les mines techécoslovaques.

Le président du gouvernement tchécoslovaque, Monsieur Zapotocky, a donné à ce propos les chiffres suivants: «L'une des plus grosses difficultés réside dans l'abstentéisme. Les heures d'absence, payées ou non, augmentent constamment. L'année dernière, le nombre des heures de travail augmenta de $2.7 \%$, et celui des absences de $21 \%$. Pendant les premiers trois mois de 1947, il y eut 55 millions d'heures d'absence; pour la même période de 1948, il y eut déjà 69.3 millions, et pour 1949, 73.5 millions. En comparaison de 1947, les absences ont augmenté en 1948 de $26 \%$ et en 1949 de $37.3 \%$.

l'absentéisme n'est pas seul en cause. D'autres phénomènes freinent considérablement la production, et avant tout, le mouvement qui s'exprime par la devise «allez lentement », et qui, sans qu'il apparaisse dans les statistiques de présence, donne pourtant son empreinte à la vitesse de la production, vitesse constamment décroissante.

Ainsi, de même que les efforts du régime communiste concernant la répartition du travail ont été contrecarrés par la résistance passive du peuple, ses efforts quant a l'augmentation de la productivité ont essuyé un échec, causé par les sentiments adverses ou tout au moins par le désintéressement du peuple quant aux buts de la politique communiste. Tout cela provient de la nature du régime qui cherche à résoudre ces deux problèmes par la même «ultima ratio » - la terreur et sa matérialisation: les camps de travaux forcés.

\section{c) Situation financière}

La troisième raison d'ordre économique contribuant à causer des embarras considérables au régime est la tendance croissante du nombre des fonctionnaires et employés d'Etat. La nécessité d'opprimer constamment l'opinion et la volonté d'une grande majorité des citoyens, entraîne inévitablement l'augmentation incessante et durable de toutes sortes de polices secrètes et de corps de sûreté armés. 
La bureaucratie, élargie encore par. l'établissement de l'administration départementale à la place de l'administration provinciale, devient de plus en plus importante et lourde. Elle accroit ainsi, non seulement la confusion qui règne dans l'administration du pays mais encore et surtout le poids tinancier qui pèse sur la caisse d'Etat. Or, cette opposition des dépenses constamment croissantes et des recettes décroissantes ne peut aboutir qu'à une crise financière pour le régime.

Le régime est donc obligé de chercher des remèdes contre cette crise. Ayant perdu toute la confiance des citoyens, même de ceux qui avaient jadis consenti à une politique communiste et qui passent aujourd'hui à une résistance passive et tacite, le régime recourt à son dernier moyen, la force. Il se procure par la force la main-d'oeuvre déficiente en imposant des travaux forcés à toute personne qui ne peut prouver sa loyauté envers le régime. Donc, le choix est abondant.

\section{Conclusion}

L'établissement des CTF en Tchécoslovaquie - il vaut mieux dire des camps de concentration - n'est donc pas un hasard ou une mesure transitoire; ce phénomène terrible est intiimement lié au régime et lui est inséparable. Les raisons politiques et économiques qui le poussent à cotte mesure extrême montrent bien nettement que l'existence des CTF est liée à tout régime communiste, .. est même son essence vitale.

Le régime dictatorial pour lequel un peuple intelligent, cultivé et politiquement évolué, ne veut pas travailler - c'est-à-dire qu'il ne veut pas aider dans la poursuite de ses buts hostiles à la démocratie et au monde démocratique - n'a pas d'autre moyen pour conserver le pouvoir que de recourir à la force.

Et la brutalité que subissent aujourd'hui les citoyens des Etats soumis au communisme, ainsi que la cause qu'ils soutiennent par leur résistance obstinée, n'est pas seulement leur propre cause, mais celle du morde civilisé tout entier. Les CTF ne menacent pas seulement les citovens malheureux de ces pays, mais quoique encore à un degré différent - aussi les hommes des pays démocratiques.

\section{Etude comparative sur: la législation de conciliation et d'arbitrage*}

\section{II - ETATS-UNIS D'AMERIQUE}

Sur le plan national, le règlement des conflits du travail relève de la loi du 23 juin sur les rapports entre le travail et le patronat ${ }^{1}$. En outre de déterminer les modalités d'accréditation des représentants des organisations ouvrières, d'obliger les em-

(1) Loi de 1947 sur les rapports entre le travail et le patronat, Série législative, 1947, E.U.A. 2, Bureau international du Travail. ployeurs à négocier de bonne foi avec les organisations ouvrières reconnues et de préciser toute une série de pratique déloyales, la loi traite également de la conciliation des différends du travail dans les industries intéressant les échanges (Titre II de la loi).

- Etude préparée par un professeur de la Faculté des sciences sociales de Laval.

La Revue des relations indụstriellegs 